\section{Prä- statt Probiotika?}

$\mathrm{N}$ achdem Probiotika die in sie gesetzten Hoffnungen in punkto Allergieprävention nicht erfüllen konnten, stehen jetzt Präbiotika auf dem Prüfstand: Dazu wurden bis zu acht Wochen alte Neugeborene, die nur ein geringes familiäres Atopierisiko aufwiesen, randomisiert entweder mit präbiotischer Formulamilch ( $n=414)$ oder einer regulären Formulanahrung ( $n=416)$ ernährt, eine Referenzgruppe von 300 Säuglingen wurde weiter gestillt. Die präbiotische Milch enthielt eine spezielle Mischung neutraler sowie aus Pektin hergestellter saurer Oligosaccharide. Bis zum ersten Geburtstag traten in der Präbiotikagruppe signifikant weni-

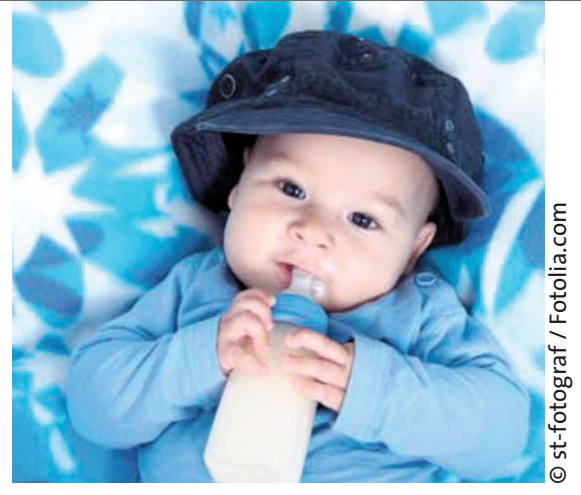

ger atopische Ekzeme auf als in der Kontrollgruppe (5,7\% vs. $9,7 \%$; $\mathrm{p}=0,04)$. Bei den gestillten Säuglingen lag die Inzidenz bei 7,3\%. Es bleibt abzuwarten, ob sich diese Unterschiede in weiteren Studien reproduzieren lassen.

uls

Grüber C et al. J Allergy Clin Immuno

2010; 126: 791-7

\title{
Kautablette mit riskantem Beigeschmack
}

B ei einer allergischen Reaktion durch Medikamente liegt der Verdacht nahe, dass der Wirkstoff der Auslöser ist - oft trifft das auch zu. Das unangenehme Nachspiel kann aber auch von den Hilfsstoffen herrühren. Das zeigt ein Fall, den Prof. Dr. Axel Schnuch aus Göttingen jetzt publiziert hat: Wegen Koliken bei einer Gastroenteritis nimmt eine Patientin Lefax $^{\circledR}$-Kautabletten ein. Fünf Minuten später juckt die Kopfhaut. Quaddeln treten am ganzen Körper auf und die Zunge wird pelzig. Eine orale Provokation mit dem Wirkstoff Simeticon - ein Silicon verläuft ergebnislos. Laut Fachinformation enthält das Präparat Fenchel-, Pfeffer- minz und Kümmelöl als Geschmackskorrigenzien. Im Pricktest stellt sich heraus, dass die Patientin gegen zahlreiche Nahrungsmittel sensibilisiert ist, darunter auch Fenchel, Pfefferminze und Kümmel.

Fazit: Bei Arzneimittelreaktionen ist nicht nur an die Wirkstoffe, sondern auch an die Hilfsstoffe als mögliche Ursache zu denken. Außerdem sollten Arzneimittel, die Nahrungsmittelbestandteile oder Pflanzenextrakte als Hilfsstoffe enthalten, einen entsprechenden Risikohinweis erhalten.

we

Arzneiverordnung in der Praxis 2010; 36 : 140-1

\section{Qualm und Ekzem gehen Hand in Hand}

$R^{a}$ aucher von mehr als 15 Zigaetten täglich weisen in multivariater Analyse eine um 40\% höhere Ein-Jahres-Prävalenz von Handekzemen auf. Dies hat eine Studie schwedischer Forscher um Birgitta Meding vom Karolinska-Institut in Stockholm ergeben. Beteiligt waren 25.851 Probanden, die im Jahr 2007 an einem nationalen Gesundheitssur-

(c) DeepReal / Fotolia.com

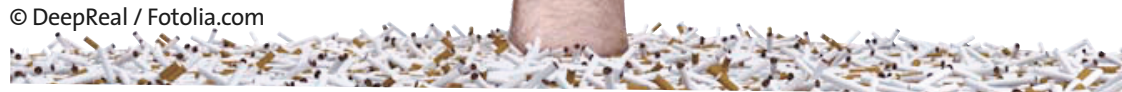

\section{Gefährliche Gerüche}

E rühlingsduft verspricht das Waschmittel, Zitronenfrische das Geschirrspülmittel. Kaum eine Seife oder ein Shampoo kommt ohne besondere Duftnote aus. Was da in die Nase steigt, ist aber bedenklich, fanden Forscher des Washington State Department of Ecology in Olympia heraus - und das nicht nur aus allergologischer Sicht. Sie untersuchten 25 parfümierte Haushaltsprodukte und fanden nicht weniger als 133 verschiedene Chemikalien, die von diesen Produkten an die Luft abgegeben wurden. Etwa ein Viertel der Emissionsstoffe gilt als potenziell toxisch oder steht im Verdacht, karzinogen zu sein. Produkte, die als ,natürlich“ oder „organisch“"vermarktet werden, machten hierbei keine Ausnahme. de

Stone $S$ et al. Environ Impact Asses 2010; 30: $380-7$

\section{Black Box Plazebo}

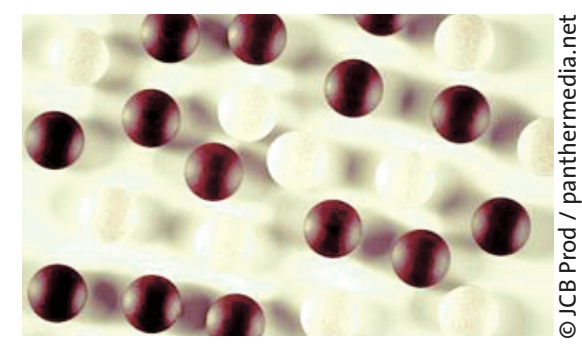

$\mathrm{n}$ der evidenzbasierten Medizin gilt die standardisierte, plazebokontrollierte Doppelblindstudie als einzig wirklich verlässlicher Wegweiser auf der Suche nach wahrer Erkenntnis. Beatrice Golomb, Medizinprofessorin aus San Diego, hat Zweifel, ob standardisiert auch wirklich standardisiert ist. In vielen Studien werde nämlich die Natur des Plazebos nicht aufgedeckt, bemängelte sie bereits vor 15 Jahren. Die Situation hat sich seitdem nicht merklich verändert: Derzeit wird bei weniger als 10\% der Publikationen angegeben, was in den Plazebopillen enthalten war. Wie aber soll man Plazeboeffekte einordnen, wenn man gar nicht weiß, um was es sich bei dem Plazebo handelt? de

Golomb BA et al. Ann Intern Med 2010; 153: 532-5 